\title{
Cold Sintering of Calcium Carbonate for Construction Material Applications
}

\author{
Mehrzad Zahabi, Aly Said,* and Ali Memari
}

Cite This: ACS Omega 2021, 6, 2576-2588

Read Online

ABSTRACT: An alternative to traditional binder-based construction materials using "cold sintering", a hydrothermal mechanism that involves subjecting the sample to simultaneous pressure and comparatively low temperatures, was explored. Ground and precipitate calcium carbonates (GCC and PCC) were used as the primary starting materials. Ordinary portland cement (OPC) and zinc oxide were studied for comparison. Compressive strength tests showed promising results from the OPC mortars with the cement mostly replaced by GCC. Scanning electron microscopy showed sintering of calcium carbonate and zinc oxide with the selection of suitable solutions. Porosity of

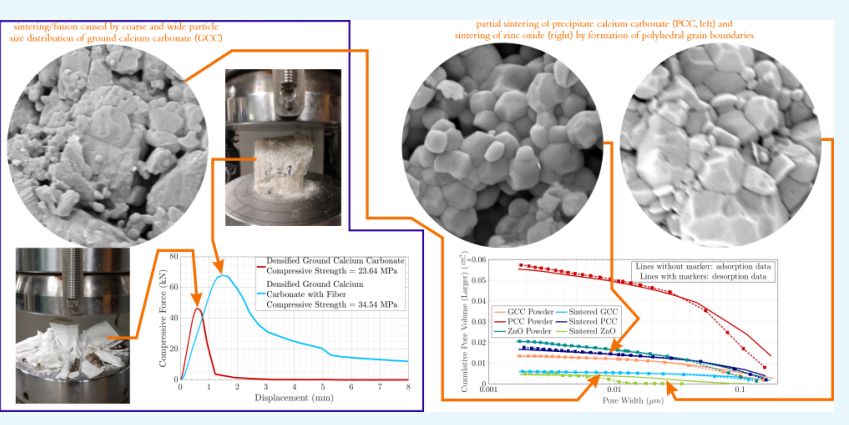
sintered samples measured by nitrogen adsorption-desorption improved considerably. These data support the feasibility of cold sintering as an alternative method for production of conventional precast construction materials.

\section{INTRODUCTION}

Ordinary portland cement (OPC) production accounts for about $7 \%$ of global $\mathrm{CO}_{2}$ emission, predominantly by the heat treatments involved $\left(\sim 1600{ }^{\circ} \mathrm{C}\right)$ and decomposition of limestone and clay. ${ }^{1}$ Other raw materials are fed into hightemperature kilns for various applications. Examples are conventional construction bricks that are manufactured by baking ground raw materials such as clay minerals (kaolin and shale) at temperatures above $1000{ }^{\circ} \mathrm{C}^{2}$ or feeding magnesiumrich dolomite to obtain sintered high-density refractory bricks after exposure to temperatures above $2000{ }^{\circ} \mathrm{C}$. ${ }^{3}$ More sustainable alternatives for traditional OPC concrete such as partial replacement with supplementary cementing materials, limestone calcined clay cements $\left(\mathrm{LC}^{3}\right),{ }^{4,5} \mathrm{CO}_{2}$ capture within ready-mix or precast concrete and masonry and synthesis of carbonate minerals, ${ }^{6}$ alternative cement clinkers such as calcium silicate clinkers sequestrating $\mathrm{CO}_{2}{ }^{7}$ and alkaliactivated and geopolymer binders have been utilized/ proposed.

$\mathrm{LC}^{3}$ is among the most promising solutions to lower carbon footprint of ordinary cement due to direct utilization of abundant materials. ${ }^{5}$ In $\mathrm{LC}^{3}$, a large portion of the ordinary cement can be replaced with untreated materials or materials processed at comparatively lower temperatures than that of ordinary cement. The most promising additives are clay and limestone due to their abundance in nature. Among the clay types, kaolinite, due to its higher pozzolanic reactivity, is the most favorable for this application when calcined at temperatures $750-850{ }^{\circ} \mathrm{C}$. Kaolinite contents as low as $40 \%$ in clay that replaces $30 \%$ of ordinary cement can furnish mechanical and durability properties comparable to or superior to concrete or mortar specimens made with ordinary cement. ${ }^{5}$ A minimum ordinary cement content needs to be maintained in the binder mixture to propel sufficient chemical reactions that provide the final concrete or mortar with acceptable mechanical properties and durability properties. Substitution of the ordinary cement by such percentages as $45 \%$ (30\% calcined clay and $15 \%$ limestone $)^{5}$ can reduce the $\mathrm{CO}_{2}$ footprint of ordinary cement largely particularly when clay quarries local to construction projects are studied and utilized.

Another alternative to traditional construction materials to achieve sustainability, and the focus of this study, could be obtained through "cold sintering". Cold sintering refers to the sintering process at comparatively lower temperatures $(\lesssim 200$ ${ }^{\circ} \mathrm{C}$ ) and sufficient pressures that can reduce the energy use associated with conventional high-temperature sintering processes. Figure S1 illustrates one example of this sample preparation. Selection of appropriate wetting agents is a critical requirement for the sintering to take place. Sourcing of the energy for the heat requirement and/or the electricity for the pressure or heat requirements of the sintering process from renewable energy sources such as concentrating solar power

Received: September 20, 2020

Accepted: December 10, 2020

Published: January 12, 2021 
(CSP) plants $^{8}$ can potentially further minimize the carbon footprint associated with precast materials such as conventional bricks and concrete masonry units (CMUs) that incorporate ordinary portland cement. Cold sintering of local materials, by the virtue of its simplicity and low equipment/ maintenance requirements, is particularly useful in remote areas to obtain construction materials such as building blocks. Desirable strengths are often obtained upon heat treatment after the press 9 but their cold sintering using suitable wetting agents can be studied.

In the literature, paste samples incorporating crystalline or amorphous fine powders into the matrix have been prepared for cold sintering. ${ }^{10,11}$ Various lubricants/solvents for this process, such as water and acids, have been explored (e.g., see Table 1 in ref 12). Studied crystalline materials include zinc oxide, vanadium pentoxide, and others, with the less watersoluble of these additionally employing a solvent other than water as the wetting agent. ${ }^{12,13}$ Hydrothermal cold sintering, a physicochemical mechanism, is caused by compressive stress concentration at contact zones of the solutes, diffusing the matter. Supersaturation caused by evaporation of the liquid phase propels the precipitation to outside of the zones and results in densification and grain boundary growth. ${ }^{12}$

For construction material applications, sintering of calcium carbonate, clay, and silica is of particular interest due to their abundance and affinity. In utilization of such raw materials, the sintering objective is to obtain composite materials through proportioning of the raw materials and incorporation of sufficient yet small doses of such binders as OPC pastes. Limestone as the primary source of ordinary portland cement is chiefly composed of calcium carbonate crystalline forms such as calcite and aragonite. Cold sintering of synthesized nanosized vaterite, another form of calcium carbonate, in aqueous and oil media has been explored and reported to have more desirable mechanical properties compared to conventional construction materials such as concrete and stone. ${ }^{14}$ It was shown that after the applied stress reached the maximum constant (creep) stress and at a given relative porosity, higher maximum creep stresses caused higher strain rate and hence higher densification. The powder starting particle size distribution (PSD) is another important factor in density improvement. In the same study, the nanoscale size of the synthesized vaterite grains significantly increased the strain rate at a creep stress of $10 \mathrm{MPa}$ and the relative porosity when compared to that of the sintered product of natural calcite with a grain size of $94 \mu \mathrm{m}$. In cold sintering of lab-synthesized nanosilica, additional water was reported to largely eliminate mesopores and hence significantly improving the densification. ${ }^{11}$ The number of macropores, however, increased, reaching several hundreds of nanometers in samples with additional water, lowering their fracture toughness and making them very brittle. The macropore presence was attributed to (1) disturbance in organized packing of the starting nanosilica particles caused by additional water and (2) possibly water being trapped in the macropores prior to the sintering.

This study examines suitability of calcium carbonate in cold sintering as the sole solid constituent or when combined with ordinary cement to form a binder. Therefore, the remainder of this introduction is focused on the fundamental properties of calcium carbonate, its sintering, and cold sintering sample preparation.

Water content and thermodynamic equilibrium affect the crystalline form of calcium carbonate. Mechanical stirring was shown to transform the calcium carbonate into more vaterite, while additional water resulted in the conversion of vaterite to calcite. ${ }^{15}$ Grinding was shown to change the $\mathrm{CaCO}_{3}$ crystal structure. ${ }^{16,17}$

Although calcium carbonate is not very stable in water, its affinity for water can vary largely depending on particle size distribution and grain coatings. Figure S2 illustrates the suspension of four grades of calcium carbonate (1 gram in 17 grams of water). They are uncoated Whiton P-30 ground calcium carbonate (GCC) (Figure 2), and three precipitate calcium carbonate (PCC) grades, uncoated Brilliant 1500 (average particle size of $150 \mathrm{~nm}$ ) (Figure 3), Silver-W, and ultrafine Viscoexel 30 (average particle size of $30 \mathrm{~nm}$ coated with fatty acids). They were obtained from Shiraishi Calcium Kaisha Ltd., Japan. Whiton P-30 GCC exhibited higher affinity for water due to coarser particle size distribution (PSD) (Figure 1) compared to the three PCC grades. The PCC

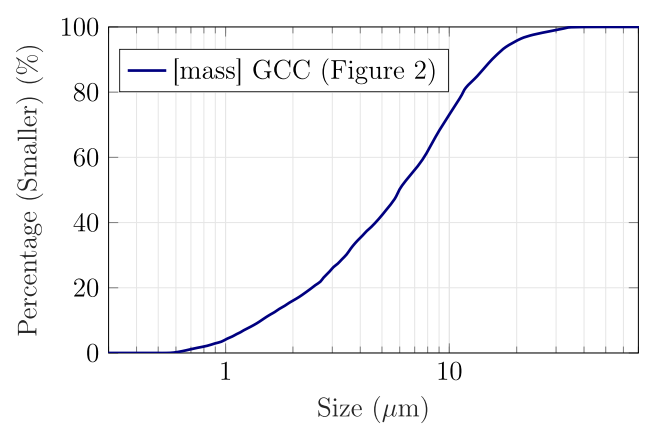

Figure 1. Particle size distribution of ground $\mathrm{CaCO}_{3}$ (Whiton P-30) utilized in the cold sintering tests. Data was provided by the manufacturer (Shiraishi Calcium Kaisha Ltd., Japan).

grades were synthesized by reaction of $\mathrm{Ca}(\mathrm{OH})_{2}$ and $\mathrm{CO}_{2}$ and exhibit more uniform PSDs. Other factors aside, owing to its finer and more uniform PSD, PCC is expected to outperform GCC in the development of regular grain boundaries under cold sintering. ${ }^{14,18}$ Howbeit, its synthesis cost/energy may not enlist it as the first alternative. Narrower and finer PSD of GCC is therefore preferred.

Another parameter in the effectiveness of cold sintering is the containment of the sample within the mold. Early and excessive escape of the liquid medium utilized in cold sintering within the mold gaps affects the density gain where notably higher pressures might be needed to gain the desired density. Use of polyvinyl pyrrolidone (PVP) adhesive as a sealant against water escape was reported to reduce the pressure needed to obtain full density from $250 \mathrm{MPa}$ for unsealed zinc oxide samples to $140 \mathrm{MPa}{ }^{19}$ The extra pressure needed for unsealed samples could partly be caused by the high friction forces developed in the gaps resulting from the transport of the liquid medium carrying the solute in the early stage of cold sintering heat/pressure application and the solute residue left in the gaps upon evaporation of the liquid medium. Therefore, a sealed design to minimize the escape in the early stage of the sintering is beneficial. The ultrafine Viscoexcel-30 PCC was originally utilized in the cold sintering tests of our study. Its repellency of water caused by the coating in addition to its fine particle size did not lead to sintered samples with sufficient cohesion. The fine particle size increased escape of the sample within gaps of the mold during the sintering, causing friction and potentially lowering transfer of the applied stress on the 


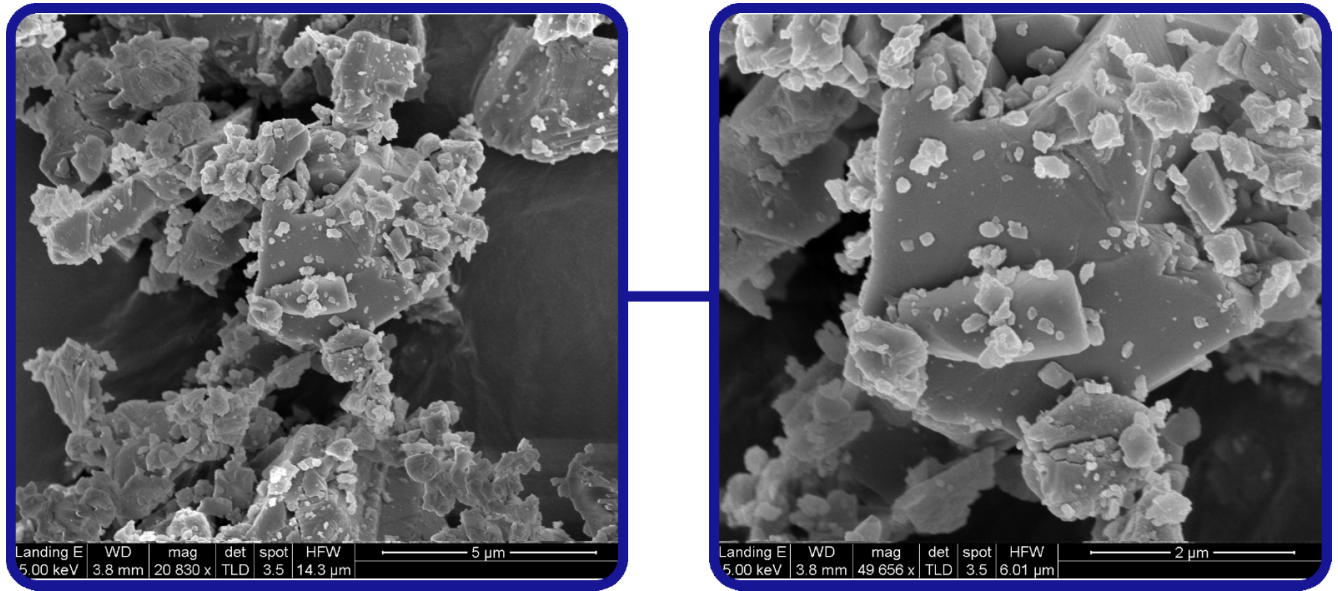

Figure 2. Uncoated GCC (Figure 1). As-received nondispersed powder was imaged.

sample. The frictional forces developed also led to higher sensitivity of the sample to circumferential cracking, forming stacked layers of dense compacts. This was observed to a lesser extent when Brilliant 1500 PCC was utilized.

$\mathrm{CO}_{2}$ facilitates solubility of limestone in water, ${ }^{20}$ and its utilization at various pressures in cold sintering of micron-sized limestone powder was reported to produce denser compacts. ${ }^{21}$ Sintering of calcite with a starting average diameter of $150 \mathrm{~nm}$ (at $450{ }^{\circ} \mathrm{C}$ under a low pressure of $2 \mathrm{MPa}$ utilizing a low dose of a mixture of $\mathrm{KF}, \mathrm{NaF}$, and $\mathrm{LiF}$ ) yielded a $99 \%$ relative density, which was more than the maximum $92 \%$ relative density in samples with a starting average diameter of $12-15$ $\mu \mathrm{m} .{ }^{22}$ Porosity of the latter did not improve despite sintering at a higher temperature of $510{ }^{\circ} \mathrm{C}$. Temperatures higher than 600 ${ }^{\circ} \mathrm{C}$ would have resulted in the decomposition of the calcite.

As discussed earlier, the sample-dependent wetting agent, the liquid used in cold sintering, is of paramount importance. Rainwater with dissolved carbon dioxide (and therefore mildly acidic) is the key factor in higher solubility of calcium carbonate in this water (the following equilibrium toward the right side) and, upon travel of the solution, leads to the formation of cave stalactites and stalagmites (reversal of the equilibrium to the left)

$$
\underset{\text { solid }}{\mathrm{CaCO}_{3}}+\mathrm{H}_{2} \mathrm{O}+\underset{\text { aqueous }}{\mathrm{CO}_{2}} \rightleftharpoons \underset{\text { aqueous }}{\mathrm{Ca}\left(\mathrm{HCO}_{3}\right)_{2}}
$$

Sulfuric acid $\left(\mathrm{H}_{2} \mathrm{SO}_{4}\right)$ and nitric acid, generated primarily by human activity through gaseous emissions of sulfur dioxide and nitrogen oxides, are major contributors to the acidity of (polluted) rainwater in the modern era. ${ }^{23}$ Dry deposition of the gaseous pollutants or wet deposition in the form of acid rain is the main cause of erosion in historic monuments and components made of limestone and marble. ${ }^{24}$ In the case of sulfuric acid, this degradation occurs by the formation of gypsum, as illustrated by the following reaction

$$
\underset{\text { solid }}{\mathrm{CaCO}_{3}}+\underset{\text { aqueous }}{\mathrm{H}_{2} \mathrm{SO}_{4}} \rightarrow \underset{\text { gypsum }}{\mathrm{CaSO}_{4}}+\underset{\text { gas }}{\mathrm{CO}_{2}}+\mathrm{H}_{2} \mathrm{O}
$$

Therefore, milder acidic solutions such as acetic acid, despite increasing the solubility of calcium carbonate by the following reaction, are less likely to be beneficial in the cold sintering of calcium carbonate. The generated calcium acetate (Ca$\left.\left(\mathrm{CH}_{3} \mathrm{COO}\right)_{2}\right)$ is more soluble in water but its decomposition to $\mathrm{CaCO}_{3}$ (one alternative to synthesize acetone) requires temperatures higher than that used in this study

$$
\begin{aligned}
& \underset{\text { solid }}{\mathrm{CaCO}_{3}}+\underset{\text { liquid }}{2 \mathrm{CH}_{3} \mathrm{COOH}} \\
& \rightarrow \underset{\text { solid }}{\mathrm{Ca}\left(\mathrm{CH}_{3} \mathrm{COO}\right)_{2}}+\underset{\text { gas }}{\mathrm{CO}_{2}}+\mathrm{H}_{2} \mathrm{O}
\end{aligned}
$$

However, the aqueous solution of acetic acid with a reported optimum molarity of $1.0 \mathrm{M}$ was shown to be highly effective in sintering of zinc oxide reaching over $90 \%$ of its theoretical density. ${ }^{25}$ Cold sintered $\mathrm{ZnO}$ was reported to reach $97 \%$ of theoretical density of $\mathrm{ZnO}\left(5.61 \mathrm{~g} / \mathrm{cm}^{3}\right)$ and to have a $40 \%$ reduction in strength (with a characteristic strength of 64 $\mathrm{MPa}$ ) compared to traditionally sintered $\mathrm{ZnO}^{26}$

On the other hand, salinity of the water improves the solubility of calcium carbonate. ${ }^{27,28}$ Aqueous solution of $\mathrm{NaCl}$ ( 0.9 weight \%) enhanced the sintering of nanovaterite possibly by increasing its solubility. ${ }^{14}$ The nanovaterite was synthesized by the precipitation reaction of sodium carbonate with calcium chloride in an aqueous solution of ethylene glycol. The conventional reaction takes the following form

$$
\underset{\text { aqueous }}{\mathrm{Na}_{2} \mathrm{CO}_{3}}+\underset{\text { aqueous }}{\mathrm{CaCl}_{2}} \rightarrow \underset{\text { aqueous }}{2 \mathrm{NaCl}}+\underset{\text { solid }}{\mathrm{CaCO}_{3}}
$$

The reverse reaction does not occur in most conditions, and an indirect method by burning of coke is utilized in the Solvay process in sodium carbonate manufacturing. ${ }^{29}$

An alkaline environment of $10 \mathrm{M}$ aqueous solution of sodium hydroxide has been reported favorable in cold (hydrothermal) sintering of calcium carbonate with the following reaction driving toward the right on the surface of the calcium carbonate grains at a higher maximum temperature of $300{ }^{\circ} \mathrm{C} .{ }^{30}$ The reaction mostly leans to the left with cold sintering at an ambient temperature without calcium hydroxide formation

$$
\underset{\text { solid }}{\mathrm{CaCO}_{3}}+\underset{\text { aqueous }}{2 \mathrm{NaOH}} \rightleftharpoons \underset{\text { aqueous }}{\mathrm{Ca}(\mathrm{OH})_{2}}+\underset{\text { aqueous }}{\mathrm{Na}_{2} \mathrm{CO}_{3}}
$$

The grain shape of the sintering product was more smooth and round, and sintered necks formed more abundantly when a higher maximum temperature of $300{ }^{\circ} \mathrm{C}$ mostly under the creep stress of $70 \mathrm{MPa}$ was utilized. ${ }^{30}$ Higher temperatures less than calcium carbonate decomposition temperature potentially enhance the sintering. $\mathrm{CaCO}_{3}$ particle coalescence was shown more pronounced in samples subjected to a stress of $650 \mathrm{MPa}$ with subsequent heating at $600{ }^{\circ} \mathrm{C}$ for $10 \mathrm{~min} .{ }^{31}$ Water-free amorphous calcium carbonate (ACC) nanoparticles sintered at 

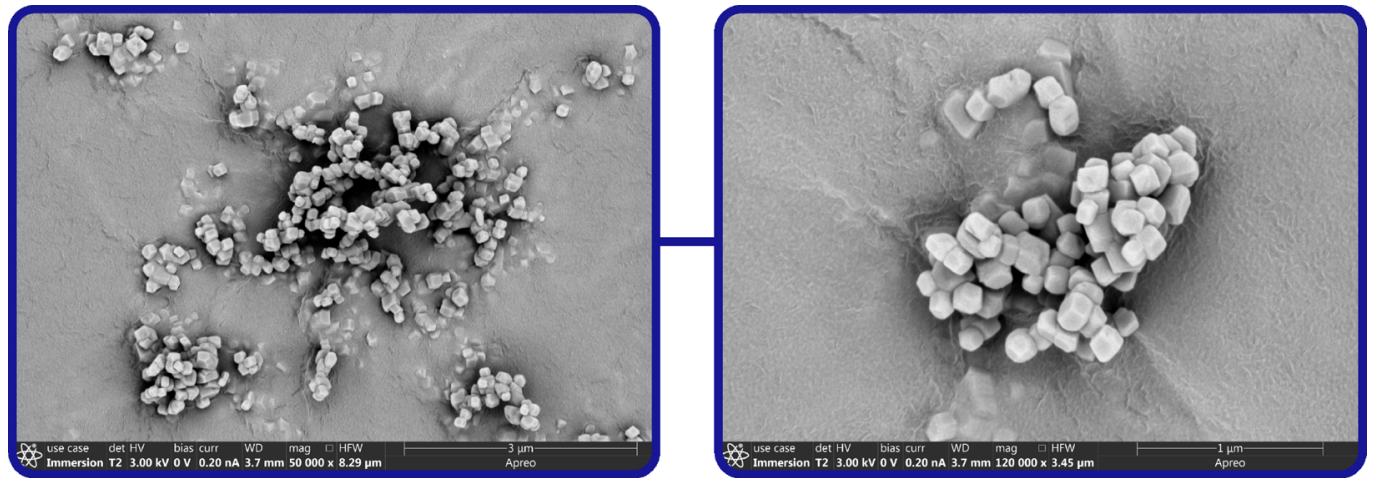

Figure 3. Uncoated PCC (average particle size: $150 \mathrm{~nm}$ ). As-received nondispersed powder was imaged.

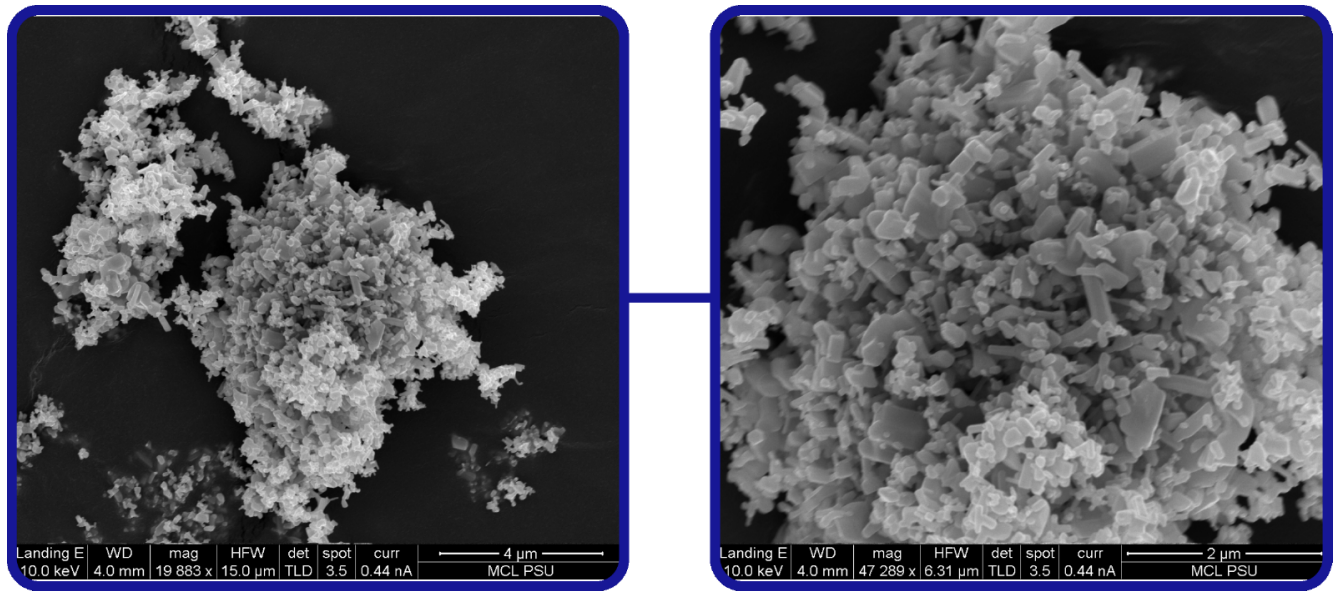

Figure 4. $\mathrm{ZnO}$ starting powder. As-received nondispersed powder was imaged.

Table 1. Mix Proportion of Samples in the Nitrogen Adsorption-Desorption (NAD) Porosimetry Study in Terms of Mass Ratio with Respect to Noncementitious Solid in Sintered/Densified Samples and to Cement in Nonsintered Samples

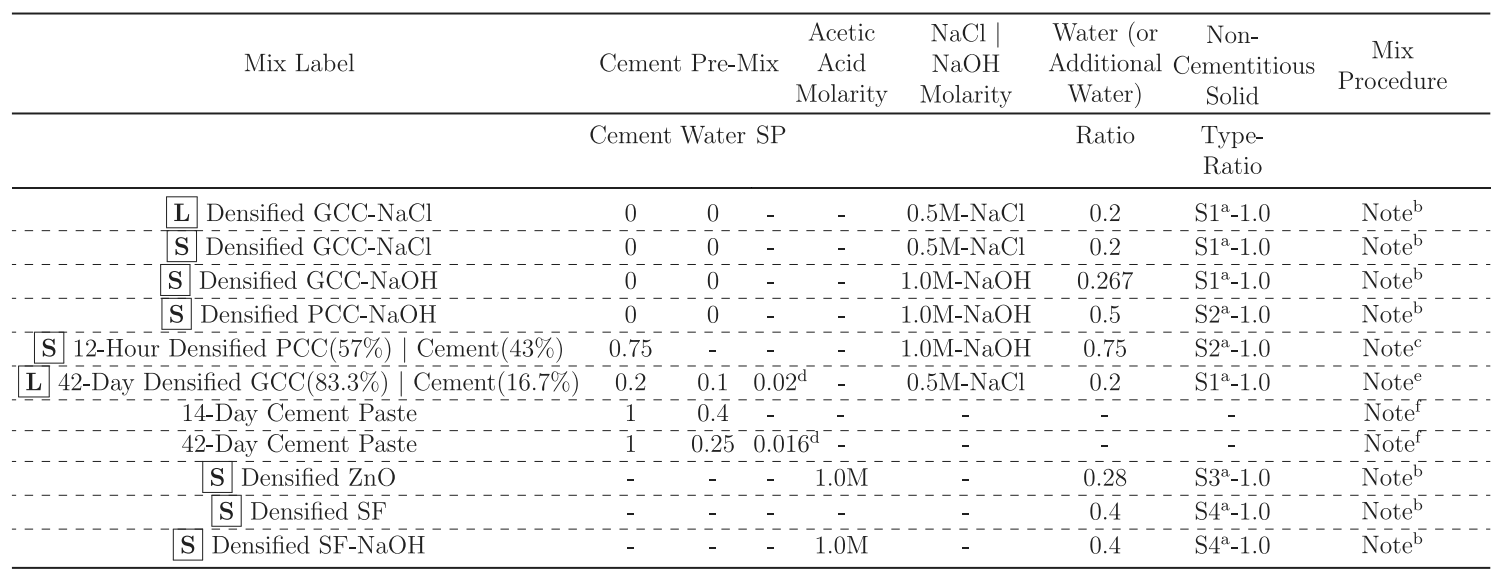

a $\mathbf{S}$ and $\mathbf{L}$ refer to densified samples prepared by the large and small molds. All samples containing cement were cured in a water bath saturated with $\mathrm{Ca}(\mathrm{OH})_{2}$ at room temperature until the test date. S1: GCC, S2: PCC, S3: $\mathrm{ZnO}$, and S4: silica fume. ${ }^{b}$ Noncementitious solid was added to the aqueous solution and ground using a mortar and pestle. ${ }^{c}$ Noncementitious solid was added to the aqueous solution and ground using a mortar and pestle. Cement was added and further ground. ${ }^{d}$ Added to the cement mix first. ${ }^{e} \mathrm{GCC}, \mathrm{NaCl}$, and water were added together originally and ground using a mortar and pestle. Additional water, superplasticizer (SP), and cement were mixed and added to the original mixture and mixed with a highshear mixer for $5 \mathrm{~min}$ at $1800 \mathrm{rpm} .{ }^{f}$ Not a sintered/densified sample. Water, superplasticizer, and cement were mixed with a high-shear mixer for 5 $\min$ at $1800 \mathrm{rpm}$.

250-400 ${ }^{\circ} \mathrm{C}$ under $20 \mathrm{MPa}$ compressive stress exhibited higher strength compared to those with lower sintering temperature. $^{32}$ Surface area reduction was reported in the sample sintered at $400{ }^{\circ} \mathrm{C}$. This was attributed to pore collapse. Solid-solid-state transformation was believed to convert ACC to aragonite and vaterite phases and, rapidly, to calcite at $400{ }^{\circ} \mathrm{C}$. 
Table 2. Mix Proportion of Samples in the Compressive Strength Study (Using the Large Mold) in Terms of Mass Ratio with Respect to Noncementitious Solid in Sintered/Densified Samples and to Cement in the Nondensified Sample

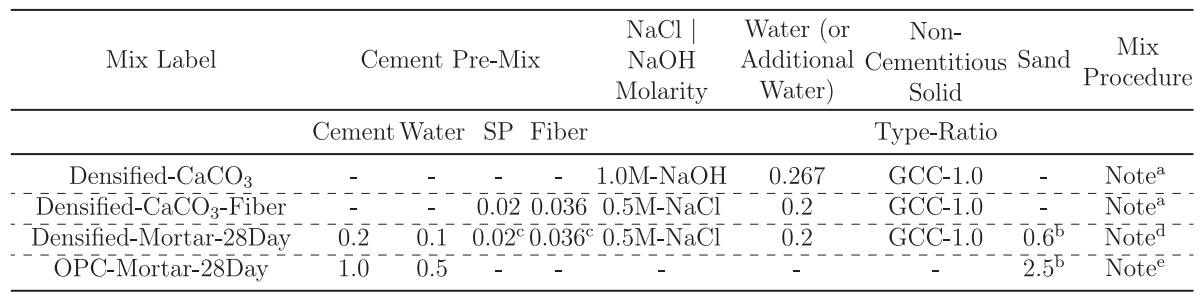

${ }^{a}$ Noncementitious solid was added to the aqueous solution and ground. ${ }^{b}$ Angular dolomitic normal-weight sand; particle size between sieves \#30 and $\# 50(297 \mu \mathrm{m} \lesssim$ particle size $\lesssim 595 \mu \mathrm{m})$. ${ }^{c}$ Added to the cement mix first. ${ }^{a} \mathrm{CaCO}_{3}, \mathrm{NaCl}$, and water were added together originally and ground using a mortar and pestle. Additional water, superplasticizer (SP), fiber, and cement were mixed and added to the original mixture and shaped into a doughy consistency. Sand was added and mixed (Figure S3). The sample was cured in a water bath saturated with $\mathrm{Ca}(\mathrm{OH})_{2}$ at room temperature until the test date. ${ }^{e}$ Not a sintered/densified sample. Water and cement were mixed with a high-shear mixer for $5 \mathrm{~min}$ at $400 \mathrm{rpm}$. Sand was added and mixed. The sample was cured in a water bath saturated with $\mathrm{Ca}(\mathrm{OH})_{2}$ at room temperature until the test date.

In our work presented herein, hydrothermal cold sintering under simultaneous pressure and comparatively low temperatures was evaluated. GCC and PCC were used as the primary starting material, and ordinary portland cement (OPC) and zinc oxide were used in some of the studies for comparison purposes. Compressive strength tests were conducted to evaluate the suitability of sintered GCC/PCC as a full or partial replacement of OPC. Gas adsorption porosimetry and scanning electron microscopy (SEM) were used to study particle coalescence and densification. This study is concluded with suggestions on scaling up the production of precast blocks through cold sintering.

\section{MATERIALS AND TEST PROTOCOLS}

2.1. Materials. All grades of calcium carbonates were obtained from Shiraishi Calcium Kaisha Ltd., Japan. Ground calcium carbonate (GCC) (Whiton P-30; Figures 1 and 2) with coarser grain sizes was utilized that provided minimal sample escape when a sufficiently small liquid content was used. The uncoated precipitate calcium carbonate (Brilliant 1500 with an average particle size of $150 \mathrm{~nm}$; Figure 3) was studied for comparison. $\mathrm{ZnO}$ (ReagentPlus $99.9 \%$ with particle size $<5 \mu$ m obtained from Sigma-Aldrich; Figure 4) and silica fume (MasterLife SF 100 obtained from BASF) were used in few of the studies, as noted in the following sections.

Angular dolomitic normal-weight sand $(297 \mu \mathrm{m} \lesssim$ particle size $\lesssim 595 \mu \mathrm{m})$ was used. Type I ordinary portland cement (OPC), polycarboxylate-based Sika Viscocrete 6100 superplasticizer (SP), BASF MasterFiber M100 fiber, ACS grade $\mathrm{NaCl}$, and $1.0 \mathrm{M}$ acetic acid and $\mathrm{NaOH}$ aqueous solutions were used in samples. The superplasticizer (surfactant) was included in some samples to evaluate its contribution to obtain more homogeneous access of the solution to the powder. In all samples, deionized water was used.

Various mix proportions were used in this study. The mix proportions and their notes are shown in Tables 1 and 2 to which the following sections refer to.

2.2. Test Protocol. SI units were used throughout this study. Two molds with sample diameters of 10 and $50.8 \mathrm{~mm}$ were used, referred to as small $([\mathbf{S})$ and large $(\mathbf{L})$ molds thereafter, respectively. A typical sample preparation procedure before placement into the large mold is shown in Figure S3. For samples with only $\mathrm{CaCO}_{3}$ or $\mathrm{ZnO}$ and aqueous solutions (without sand or fiber), a low solution-to-powder ratio was used and the ground samples did not have the doughy consistency shown in the figure. The ground mixtures were compacted with a Delrin rod in few layers after placement in the mold. Fibers and superplasticizer provide the mixture with a doughy consistency. No compaction was performed on the doughy samples. A plastic bowl was used in the preparation of the samples for the large mold ( 50 gram $\leq$ sample mass $\leq 350$ grams). An agate mortar and pestle was used in the preparation of samples for the small mold with solution-to-powder ratio varying, as described in the following sections.

Utilizing the large mold, the sample was placed in a press mold at room temperature. The mold was machined from a prehardened steel. It was placed inside of a cylindrical Watlow ceramic fiber heater $(1500 \mathrm{~W}$ at $120 \mathrm{~V})$ and subjected to heat and compression. The heater had enough watt density to reach the maximum temperature $\left(150{ }^{\circ} \mathrm{C}\right)$ within $10 \mathrm{~min}$. A schematic of this test is shown in Figure S1. The heater and the mold are shown in Figures S4 and S5, respectively.

A thin layer of release agent oil was placed in the full height of the inner diameter of the tube of the large mold. The sample was placed in the mold and heated to the maximum temperature $\left(150{ }^{\circ} \mathrm{C}\right)$. The uniaxial load was slowly increased to the maximum load $(450 \mathrm{kN}$ ) within $5 \mathrm{~min}$ (using a $530 \mathrm{kN}$ compression machine) after the outside temperature of the mold reached $120{ }^{\circ} \mathrm{C}$. This maximum load was maintained for 90 min after which the mold was slowly unloaded. The maintained maximum load applied an axial stress of $230 \mathrm{MPa}$ on the partially confined sample with a diameter of $50.8 \mathrm{~mm}$. The assembly was set to cool to room temperature, and the sample was removed from the press mold using a plunger and additional support pieces underneath the mold. Mostly calcium carbonate samples with or without cement were utilized using this mold from which undamaged compacts were obtained. In the sample removal stage and for some samples, a force as high as $30 \%$ of the maximum load of $450 \mathrm{kN}$ was needed to overcome the friction caused by the sample escape within the gap between the press rod plunger/stepped base support and the interior face of the tube. This escape was inevitable despite acceptable mold precision machining (down to $0.02 \mathrm{~mm}$ in gap).

Utilization of the small mold, due to the ease of working with the small sample volumes, allowed higher control over sample preparation by the mortar and pestle grinder and hence more homogeneous access of the solution to powder. Various thermomechanical regimes were conducted that are discussed later. Figure S6 shows the small mold. 
In all samples subjected to cold sintering, the temperature vs time profile was controlled by the PID controller and the thermocouples, as shown in Figures S1, S4, and S6. Application of the force was not sophisticated. The load was applied using the load frame shown in Figure S1 and was manually/visually monitored by synchronizing with the temperature profile. Therefore, the stress profiles are not exact. This inaccuracy, however, is believed to affect the results slightly. The stress values were obtained by dividing the force by cross-sectional areas of the samples. Diameters of the samples obtained from the large and small molds were 50.8 and $10 \mathrm{~mm}$, respectively.

2.3. Material Characterization and Definitions. 2.3.1. Scanning Electron Microscopy (SEM). An FEI Nova NanoSEM 630 or Apreo SEM with field emission guns was used in secondary electron microscopy of samples. They were sputter-coated with 7-10 nm of iridium using a Leica EM ACE600 coater. Hydration of samples with ordinary portland cement was stopped using multiple isopropanol exchange within a day. As shown in Figure 5, various stress-temperature profiles were used for the SEM study.

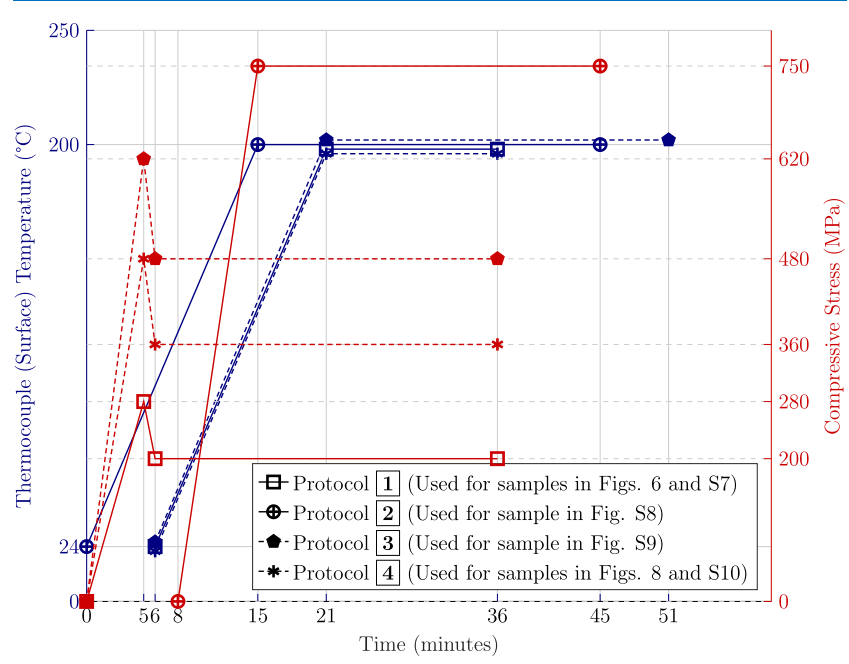

Figure 5. Approximate compressive stress and average temperature profiles of PCC samples (prepared by the small mold) for the SEM and NAD studies.

2.3.2. Nitrogen Adsorption-Desorption (NAD). Pore volume distribution of samples was studied using nitrogen adsorption-desorption (NAD), and Brunauer-Emmett-Teller (BET) surface areas were calculated. Mix proportions of samples are shown in Table 1. Sintered/densified samples prepared by the large and small molds are marked with $\mathbf{L}$ and $\mathbf{S}$, respectively. The stress-temperature profile of $\mathbf{S}$ samples was protocol $\mathbf{1}$ in Figure 5. Samples that contained cement were immersed after the test in a water bath at room temperature saturated with $\mathrm{Ca}(\mathrm{OH})_{2}$. The sample age expressed in the mix labels refers to the time hydration was stopped after the immersion.

To remove the physically bound water and stop the hydration in samples with cement, solvent (isopropanol) exchange was utilized. This was done to ensure removal of any water remaining in the cement paste microstructure. The samples were ground by an agate mortar and pestle 3-4 days prior to the measurement. With the exception of starting powders, $0.8-1.2$ grams of sample particles passing sieve No.
16 and retained on sieve No. $50(297 \mu \mathrm{m} \lesssim$ particle size $\lesssim$ $1190 \mu \mathrm{m}$ ) were transferred to tubes and $40 \mathrm{~mL}$ of cool (at 3 ${ }^{\circ} \mathrm{C}$ ) isopropanol was added. The low temperature helps preserve the microstructure of cement hydrates at a higher degree. ${ }^{33}$ The tubes were shaken and let rest for at least $12 \mathrm{~h}$ in a temperature-controlled environment at $3{ }^{\circ} \mathrm{C}$. Isopropanol was discarded, and the samples were placed in a vacuum desiccator for at least 2 days to remove the remaining isopropanol and physically bound water and sealed. For samples without cement, a short isopropanol exchange at room temperature followed by vacuum desiccation was done. For degassing (to remove physically adsorbed gas from the surfaces and water from the pores), samples with cement were placed in the NAD measurement equipment (Micromeritics ASAP 2020) glass tube and under vacuum at $40{ }^{\circ} \mathrm{C}$ for at least 24 h. For samples without cement, the temperature and duration were $80{ }^{\circ} \mathrm{C}$ and $5 \mathrm{~h}$, respectively. The vapor evacuation was continued until the pressure dropped to less than $5 \mu \mathrm{mHg}$. For the analysis, the sample tube was placed in liquid nitrogen at a cryogenic temperature $\left(-196{ }^{\circ} \mathrm{C}\right)$. A filler tube was used in both degassing and measurement to minimize nitrogen consumption.

2.4. Compressive Strength. Compressive strength of cylindrical samples from the large mold (sample diameter: 50.8 $\mathrm{mm}$ ) with partial or full replacement of ordinary portland cement (OPC) by calcium carbonate was evaluated. The samples' mix proportions are shown in Table 2. A load rate of $445 \mathrm{~N} / \mathrm{s}$ was utilized. This load rate is half of the minimum recommended value $(900 \mathrm{~N} / \mathrm{s})$ of ASTM C109 for mortar cube compression tests and expected to underestimate the ultimate load given the slower rate. It is close to the recommended value $(0.25 \mathrm{MPa} / \mathrm{s})$ of ASTM C39 for cylindrical concrete specimens and caused the samples to fail within reasonable proximity to but longer than $1 \mathrm{~min}$ recommended by ASTM standards on ceramic materials (ASTM C773 and C1424). It is believed the utilized load rate is a reasonable value.

\section{RESULTS AND DISCUSSION}

3.1. Scanning Electron Microscopy (SEM). 3.1.1. Precipitate $\mathrm{CaCO}_{3}(\mathrm{PCC})$. Few solutions were explored in this study for the sintering of the PCC (Figure 3). Approximate stress-temperature profiles are shown in Figure 5. The small mold was used for these samples, allowing higher control in sample preparation and more homogeneous access of the solution to the PCC grains since sample volumes significantly smaller than those for the large mold were used.

The PCC sample prepared by $\mathrm{NaOH}$ solution had the same constituents and mix procedure as those of the sample with mix label " $\mathbf{S}$ Densified PCC-NaOH" in Table 1. As shown in Figure 6, this solution outperformed other solutions in sintering of the PCC by the formation of polyhedral grains, compared to the cubic shape of the starting powder. This polyhedral grain boundary formation was not observed when other wetting agents (solutions) were utilized, as shown by SEM images discussed later in this section. It is believed that a higher molarity of $\mathrm{NaOH}$ would have propelled a more compact sintered product, similar to that of $\mathrm{ZnO}$, discussed later in this section. There is increased potential for the consumption of $\mathrm{CaCO}_{3}$ grain surfaces per eq 5 at $300{ }^{\circ} \mathrm{C}$ but this reaction is believed to be minimal in the densified PCC sample due to the small solution-to-solid ratio and the utilized 


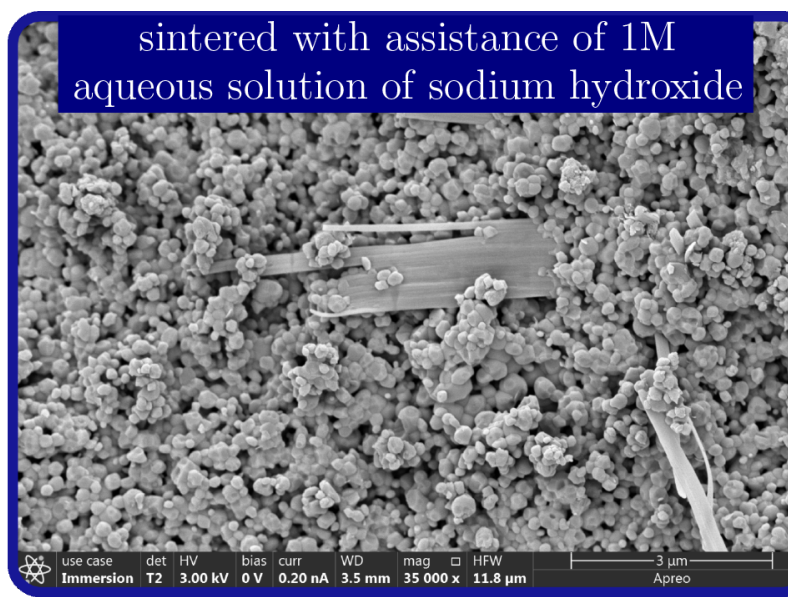

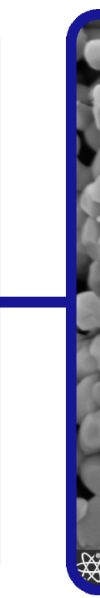
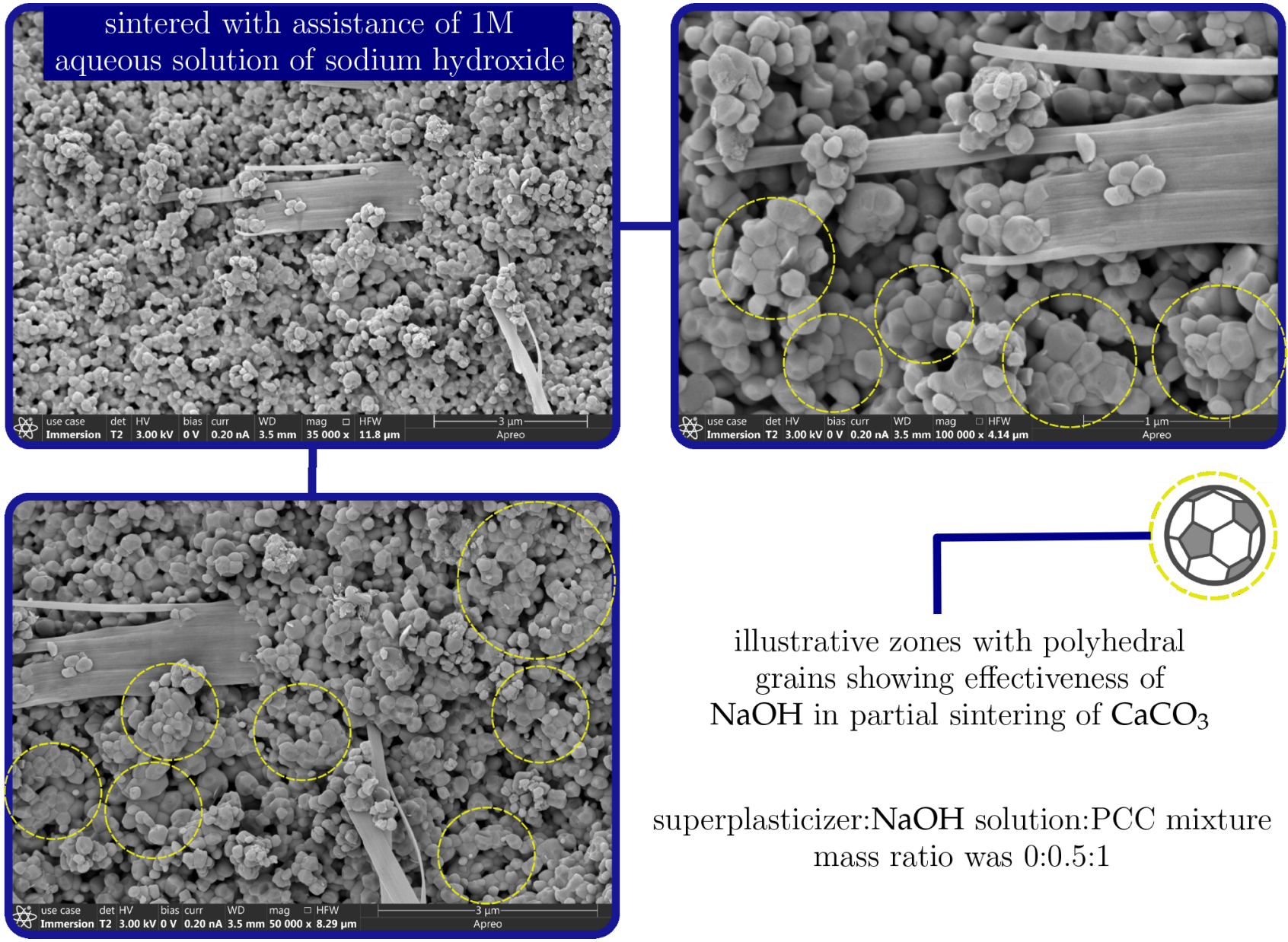

illustrative zones with polyhedral grains showing effectiveness of $\mathrm{NaOH}$ in partial sintering of $\mathrm{CaCO}_{3}$

superplasticizer: $\mathrm{NaOH}$ solution:PCC mixture mass ratio was 0:0.5:1

Figure 6. Partially sintered PCC with the assistance of $\mathrm{NaOH}$ solution. Stress-temperature profile: protocol 4 in Figure 5.

lower temperature of $200{ }^{\circ} \mathrm{C}$. Reduction of the observed pores can be achieved with other thermomechanical regimes and more certainly by isostatic stresses, utilizing cold isostatic press (CIP) or hot isostatic press (HIP) setups.

Figure S7 shows the effect of ordinary portland cement (OPC) on densification of PCC grains. Alkali ions $\mathrm{Na}^{+}$and $\mathrm{K}^{+}$ in cement pore solution can potentially have similar effects as that of $\mathrm{NaOH}$ solution in improvement of the sintering where $\mathrm{CaCO}_{3}$ is supplemented with a small dose of ordinary cement. The mixture was placed in the mold after grinding for $15 \mathrm{~min}$. Stress-temperature profile was protocol $\mathbf{1}$ in Figure 5. It appears that the consumption of water from $\mathrm{NaOH}$ solution by cement reduced sintering efficacy of the PCC grains. A small content of cement hydrates, ettringite or calcium-silicatehydrate $(\mathrm{C}-\mathrm{S}-\mathrm{H})$, is observed as a result of early water evaporation, and the hydration reactions of OPC were minimal. No EDS data was collected to distinguish between the two cement hydrates but the needles resemble ettringite. Lengthening the period between the sample mixture and the heat/stress application to few hours might be one strategy to allow the formation of more abundant $\mathrm{C}-\mathrm{S}-\mathrm{H}$. The obtained matrix of this sample, however, is compact upon an SEM survey of various areas across the sample. From the standpoint of OPC, there is a very low possibility for generation of portlandite $\left(\mathrm{Ca}(\mathrm{OH})_{2}\right)$ as the sintering test duration was less than $1 \mathrm{~h}$ during which water evaporated. Portlandite generally forms after a few hours. ${ }^{34}$ The reaction of $\mathrm{NaOH}$ with the
PCC might have produced a small amount of $\mathrm{Ca}(\mathrm{OH})_{2}$ (eq 5). No signature portlandite plates were observed in SEM imaging. High stress applied is another reason that might have prohibited the growth of portlandite crystals within the matrix. The low portlandite content makes the binder resistant to carbonation.

A $1 \mathrm{M}$ aqueous solution of $\mathrm{NaCl}$ (Figures S8 and S9) or acetic acid (Figure S10) does not seem to be as effective in sintering of PCC. Superplasticizer was used in the three samples shown in Figures S8-S10. Its higher dose in the latter two is believed to have facilitated a more homogeneous access of the solution to $\mathrm{CaCO}_{3}$ particles and led to high densification. Superplasticizer addition nevertheless did not help regular grain boundary development when compared to the PCC sample with $\mathrm{NaOH}$ solution only (Figure 6). Low superplasticizer dosage in Figure S8 did not lead to PCC grain deformation observed in samples with higher superplasticizer dosage (Figures S9 and S10). Also, maintenance of the ultrahigh stress of $750 \mathrm{MPa}$ in the sample in Figure S8 was not effective in the formation of grain boundary. The combined effect of a lower stress and a suitable solution (such as $\mathrm{NaOH}$ solution) with the temperature increase taking place after an existing compressive stress appears to be the most effective means in the uniaxial sintering of PCC. In the case of the sample with acetic acid (Figure S10), eq 3 easily develops at room temperature and mostly during the sample preparation and prior to placement in the mold but most interior zones of 


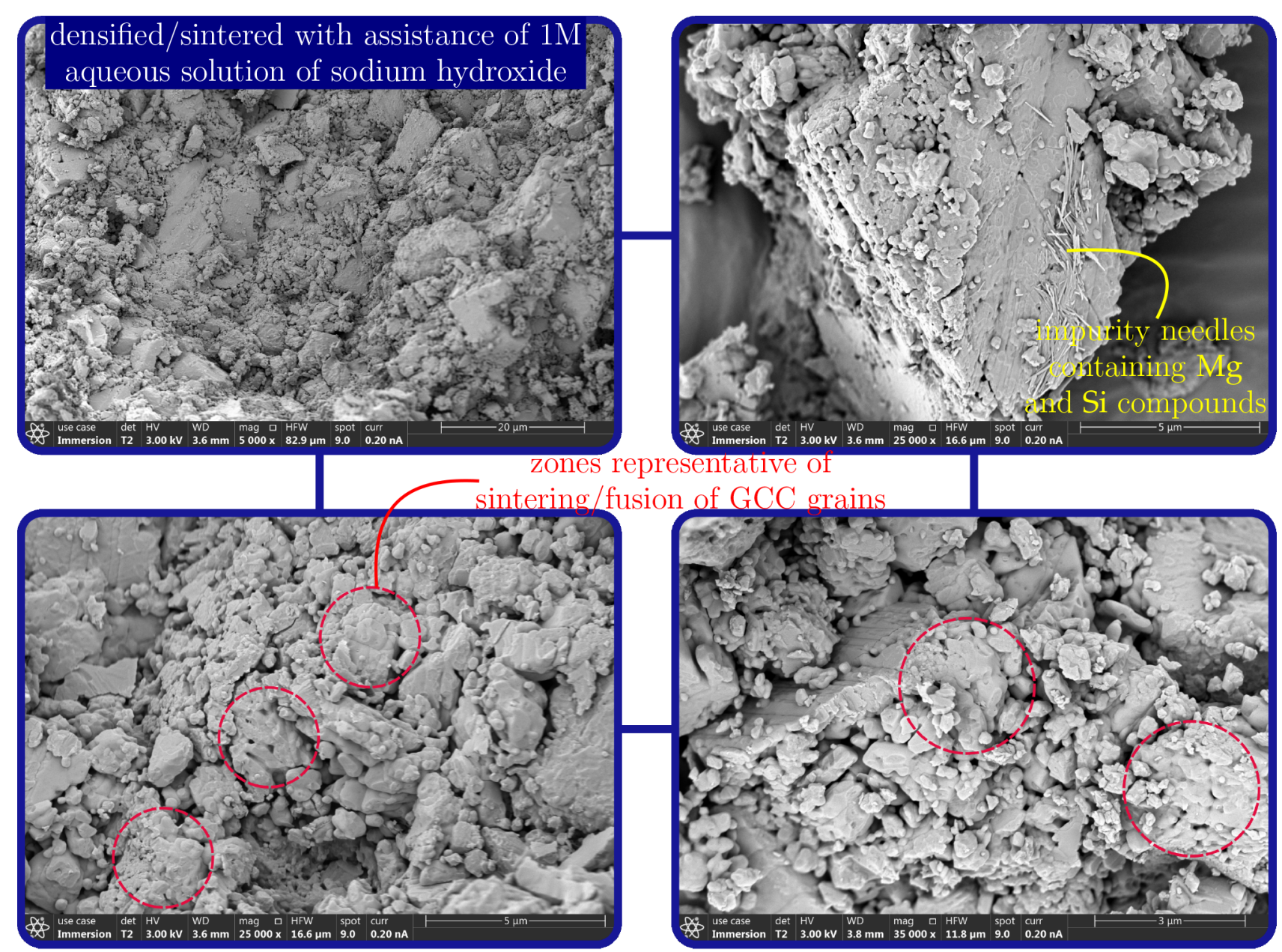

superplasticizer: $\mathrm{NaOH}$ solution:GCC mixture mass ratio was 0:0.25:1

Figure 7. Densified/sintered GCC with the assistance of $\mathrm{NaOH}$ solution. Stress-temperature profile: protocol $\mathbf{1}$ in Figure 5.

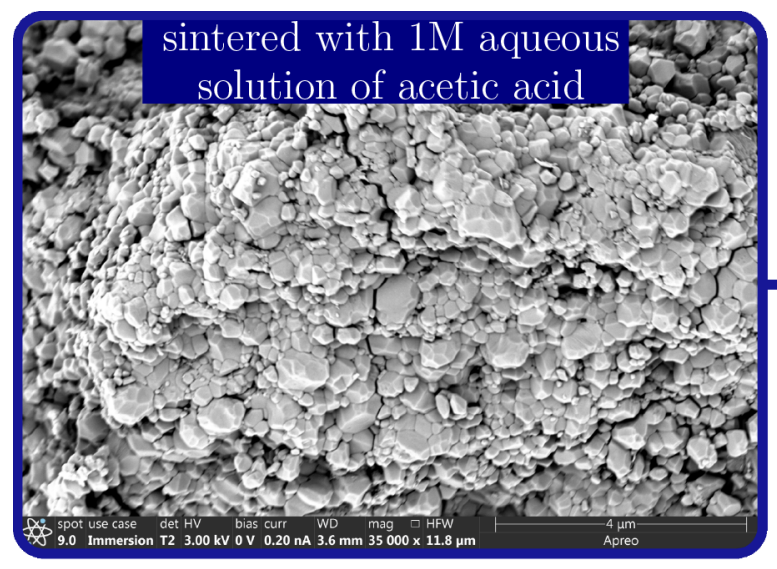

superplasticizer:acetic acid solution: $\mathrm{ZnO}$ mixture mass ratio was 0.03:0.2:1

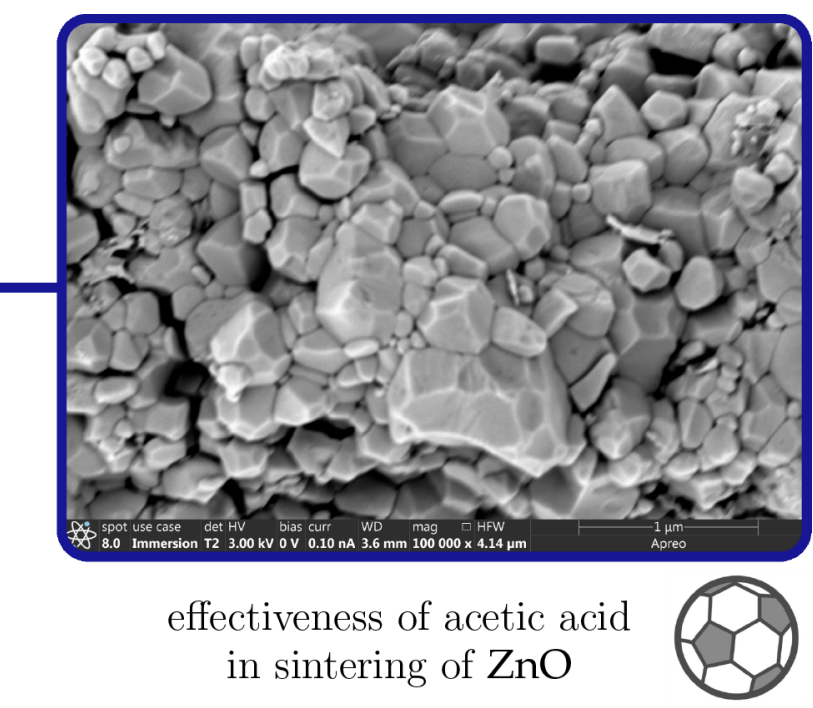

Figure 8. Sintered $\mathrm{ZnO}$ with acetic acid solution and superplasticizer. Stress-temperature profile: protocol $\mathbf{4}$ in Figure 5.

the $\mathrm{CaCO}_{3}$ grains are not consumed due to the low solutionto-solid ratio.

3.1.2. Ground $\mathrm{CaCO}_{3}$ (GCC). SEM images of the starting GCC powder are shown in Figure 2. Figure 7 illustrates the lower effectiveness of $\mathrm{NaOH}$ solution in the development of regular (straight) grain boundaries in the GCC sample when compared to that of the PCC sample (Figure 6), predominantly due to its coarser, irregular, and nonuniform particle sizes. Grain boundary growth was nonetheless observed in areas with smaller grains. The sample was 
structurally stronger than sintered PCC samples due to its higher densification, as discussed in the Nitrogen AdsorptionDesorption (NAD) section. Cheaper production of GCC makes it an attractive option, and addition of aggregates and a small supply of ordinary cement can further improve the structural soundness, as showcased in the Compressive Strength section of the Results and Discussion section.

3.1.3. Zinc Oxide ( $\mathrm{ZnO})$. Figure 4 illustrates the starting $\mathrm{ZnO}$ powder. The effectiveness of $1 \mathrm{M}$ acetic acid solution in the sintering of $\mathrm{ZnO}$ is shown in Figure 8. Its stresstemperature profile was protocol $\mathbf{1}$ in Figure 5. Similar results are reported in the literature with smaller sustained stresses. ${ }^{25}$ Superplasticizer was used to lower the solution-tosolid ratio to 0.2 but typically, the acetic acid solution without superplasticizer is adequate as the wetting agent since its crystals give sufficient adhesion to the matrix within a few minutes. The utilized high stress caused the cracking along developed grain boundaries shown in Figure 8. Lower stresses can lead to similar sintering ${ }^{25}$ without fracture.

Figures S11 and S12 show the inefficacy of the sintering due to the combined effect of a higher degree of solution inhomogeneity in the mixture, use of the large mold, and the temperature reaching its $150{ }^{\circ} \mathrm{C}$ maximum before a $280 \mathrm{MPa}$ stress was sustained for $1.5 \mathrm{~h}$.

3.2. Nitrogen Adsorption-Desorption (NAD) Porosimetry. NAD pore size distributions by the Barrett, Joyner, and Halenda $(\mathrm{BJH})$ method are shown in Figures 9 and 10.

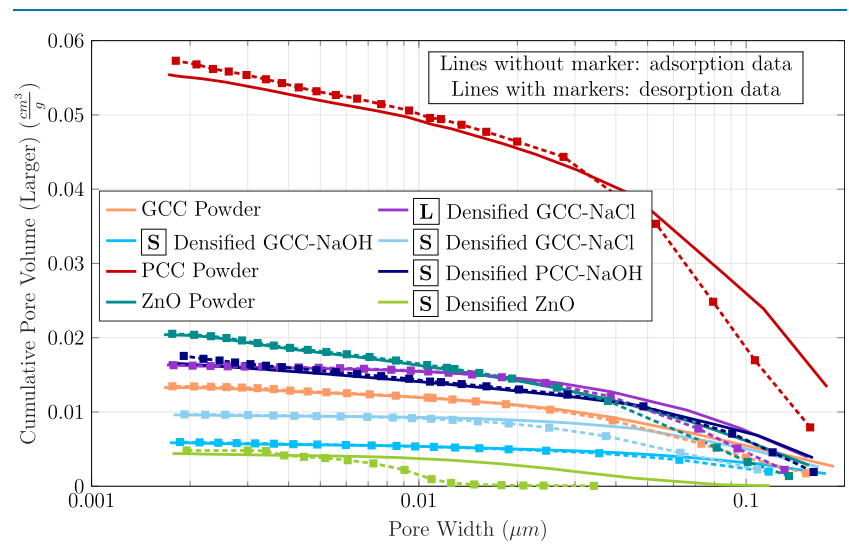

Figure 9. Nitrogen adsorption-desorption (NAD) porosity distribution of sintered/densified samples using adsorption or desorption (see the legend) isotherms (BJH method with Harkins and Jura: Faas correction). $\mathbf{L}$ and $\mathbf{S}$ refer to densified samples prepared by the large and small molds, respectively.

The BET surface areas are shown in Tables 3 and 4 . Comparing adsorption (or desorption) data among samples, the sintered/densified GCC sample with the small mold shows significant improvement when compared to the manufacturerprocessed raw GCC powder (as-received) and the sample prepared by the large mold. This is a result of a combined effect of $\mathrm{NaOH}$ solution, the small mold utilization, and, possibly, higher stress and temperature in the former. This sample had a density of $2.36 \mathrm{~g} / \mathrm{cm}^{3}$, reasonably high compared to calcite theoretical density $\left(2.71 \mathrm{~g} / \mathrm{cm}^{3}\right)$. This can be further improved by isostatic press or subsequent heat treatment more than the utilized mold surface temperature of $200{ }^{\circ} \mathrm{C}$. The GCC sample prepared by the small mold and the $\mathrm{NaCl}$ solution had an acceptable densification as well (density: 2.32

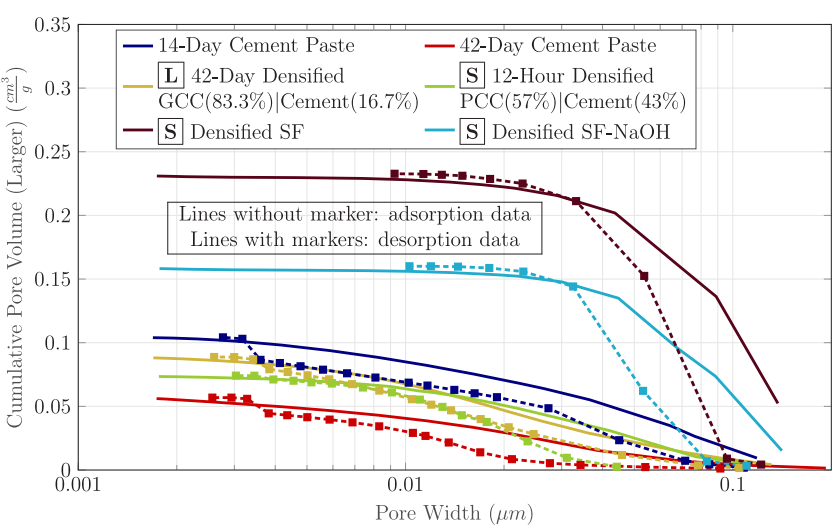

Figure 10. Nitrogen adsorption-desorption (NAD) porosity distribution of sintered/densified and cement paste samples using adsorption or desorption (see the legend) isotherms (BJH method with Harkins and Jura: Faas correction). $\mathbf{L}$ and $\mathbf{S}$ refer to densified samples prepared by the large and small molds, respectively.

$\left.\mathrm{g} / \mathrm{cm}^{3}\right)$. The GCC sample prepared by the large mold shows higher porosity across the full range of pore size compared to the raw powder. This is believed to be caused chiefly by the difficulty associated with preparing a large specimen and achieving an adequate level of homogeneity since the prepared sample mass was about 300 grams, significantly larger than the sample used in the small mold.

PCC samples were prepared by the small mold only. Densification of the PCC was pronounced after sintering with $\mathrm{NaOH}$ solution. This sample was not as dense as the GCC sample with $\mathrm{NaOH}$ solution. It is believed that the grain boundary growth in the former (Figure 6) grouped PCC grains into aggregates, leaving behind large pores. This could also have been facilitated by evaporation of the solution remnants, leaving behind the pores. This, however, was not observed in the GCC sample (Figure 7) despite irregular boundaries. The wide particle size distribution is believed to have caused their close-packing and higher densification.

The porosities of traditionally prepared (not sintered/ densified) cement paste samples were compared to those of sintered/densified samples. As expected, the 42-day-old cement paste with a mix water-to-cement ratio of 0.25 (Table 1) is less porous than the 14-day-old cement paste sample with a mix water-to-cement ratio of 0.4 due to the continuous production of additional cement hydrate phases over time, the low water-to-cement ratio, and the use of superplasticizer. Porosity of the 42-day-old cement paste sample can slightly decrease over time by further hydration. These two samples were studied to capture a reasonable range of the porosity of early-age cement pastes. The 42-day-old densified sample made of $83.3 \%$ GCC and $16.7 \%$ cement as the solid content exhibited higher porosity compared to the 42-day-old cement paste, due to the aforementioned causes. The 12-hour-old sample made of 57\% PCC and $43 \%$ cement as the solid content, however, had denser microstructure compared to the 14-day-old cement paste. It is expected that utilization of more homogeneous sintered samples with a combination of PCC grades or finer GCC particles and cement will result in samples with higher compaction (Figure S7) when compared to that of aged cement paste.

Sintering of $\mathrm{ZnO}$ using acetic acid solution is shown to reduce the porosity and BET surface area. This improvement is more pronounced compared to sintering of calcium carbonate 
Table 3. Surface Area of Sintered/Densified Samples Measured by Nitrogen Adsorption-Desorption

\begin{tabular}{|c|c|c|c|c|c|c|c|c|}
\hline & $\begin{array}{l}\frac{\dot{0}}{0} \\
0 \\
0 \\
0 \\
0 \\
0 \\
0\end{array}$ & 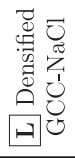 & 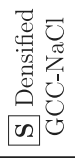 & 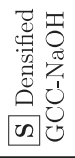 & $\begin{array}{l}\overrightarrow{0} \\
\overrightarrow{0} \\
0 \\
0 \\
0 \\
0 \\
0 \\
0\end{array}$ & 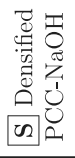 & $\begin{array}{l}\frac{5}{0} \\
\vdots \\
0 \\
0 \\
0 \\
0 \\
\text { ज. }\end{array}$ & 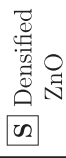 \\
\hline BET Surface Area $\left(\mathrm{m}^{2} / \mathrm{g}\right)$ & 2.49 & 6.16 & 1.13 & 1.25 & 12.56 & 5.01 & 6.46 & 2.02 \\
\hline t-P $-\bar{t}$ External Surface Area $\left(\mathrm{m}^{2} / \mathrm{g}\right)$ & $\overline{2} . \overline{2}$ & $-7 . \overline{9} 2$ & $1 . \overline{5} \overline{4}$ & 1.39 & 12.58 & 6.07 & $\overline{5} . \overline{5} \overline{8}$ & $1.9 \overline{5}$ \\
\hline $\begin{array}{l}\text { BJH }^{-} \text {Adsorption cumulative surface } \\
\text { area }\left(\mathrm{m}^{2} / \mathrm{g}\right) \text { of pores }\end{array}$ & 2.20 & 6.08 & 1.01 & 0.98 & 10.66 & 4.08 & 5.77 & 1.31 \\
\hline $\begin{array}{l}\text { BJH Desorption cumulative surface } \\
\text { area }\left(\mathrm{m}^{2} / \mathrm{g}\right) \text { of pores }\end{array}$ & 2.41 & $\begin{array}{c}--- \\
6.73\end{array}$ & 1.49 & 1.14 & 12.50 & 5.04 & 5.88 & 1.98 \\
\hline
\end{tabular}

${ }^{a}$ Pore widths between 17 and $3000 \AA$.

Table 4. Surface Area of Sintered/Densified and Cement Paste Samples Measured by Nitrogen Adsorption-Desorption

\begin{tabular}{|c|c|c|c|c|c|c|}
\hline & 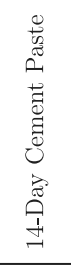 & 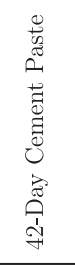 & 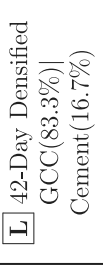 & 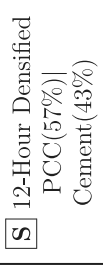 & 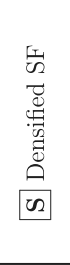 & 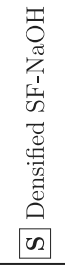 \\
\hline BET Surface Area $\left(\mathrm{m}^{2} / \mathrm{g}\right)$ & 23.57 & 21.27 & 26.16 & 16.86 & 22.31 & 18.54 \\
\hline t-P $\bar{P}$ lot External Surface Area $\left(\mathrm{m}^{2} / \mathrm{g}\right)$ & $\overline{25.74}$ & $\overline{2} \overline{3} . \overline{7} \overline{4}$ & $\overline{2} \overline{8} . \overline{9} \overline{6}$ & $\overline{18} . \overline{5}$ & 20.18 & $15 . \overline{9} 8$ \\
\hline $\begin{array}{l}\mathrm{BJH}^{-} \text {Adsorption cumulative surface } \\
\text { area }\left(\mathrm{m}^{2} / \mathrm{g}\right) \text { of pores }\end{array}$ & 26.24 & 21.48 & 28.05 & 17.76 & 18.50 & 14.01 \\
\hline $\begin{array}{c}\text { BJH Desorption cumulative surface } \\
\text { area }\left(\mathrm{m}^{2} / \mathrm{g}\right) \text { of pores }\end{array}$ & 43.45 & 30.57 & 35.94 & 25.32 & 22.55 & 17.61 \\
\hline
\end{tabular}

${ }^{a}$ Pore widths between 17 and $3000 \AA$.

samples, showing higher efficiency of acetic acid solution in sintering of $\mathrm{ZnO}$ (Figure 8) than $\mathrm{NaOH}$ solution in sintering of calcium carbonate (Figures 6 and 7). Sintered $\mathrm{ZnO}$ had a density of $5.4 \mathrm{~g} / \mathrm{cm}^{3}, 95 \%$ of the $\mathrm{ZnO}$ theoretical density of $5.67 \mathrm{~g} / \mathrm{cm}^{3}$, whereas PCC sintered with $\mathrm{NaOH}$ solution obtained $87 \%$ of calcite's theoretical density, as described earlier.

$\mathrm{NaOH}$ solution is shown to be more effective in densification of silica fume when compared to the densified sample with water only. Their porosity is large compared to the 14-day-old cement paste. Their packing when combined with cement and calcium carbonate can improve, enlisting them as potential additives in the binder.

3.3. Compressive Strength. Compressive strength results of samples from the large mold are shown in Figure 11. Samples with a sufficient height of twice as much as the diameter were not obtained due to mold size limitations. It is shown that the GCC sample sintered/densified with the assistance of $\mathrm{NaOH}$ solution and without fiber reinforcement had a strength of $23.6 \mathrm{MPa}$. This result is quite promising given no parametric study, including temperature and stress profiles, was conducted to maximize the strength. More homogeneous sample preparation methods by mechanical mixers and improvement in the utilized cold sintering setup can capture the onset of sintering, resulting in more desirable mechanical properties, suitable for structural applications.

Fiber inclusion enhanced the strength to $34.5 \mathrm{MPa}$ in the sintered/densified GCC sample. Figure 12 illustrates the failure types of the samples with and without fiber. A sample with fiber had higher postpeak capacity and ductility. $\mathrm{NaCl}$ solution was used in the sample with fiber. It is believed that $\mathrm{NaOH}$ solution improves the strength slightly. The low

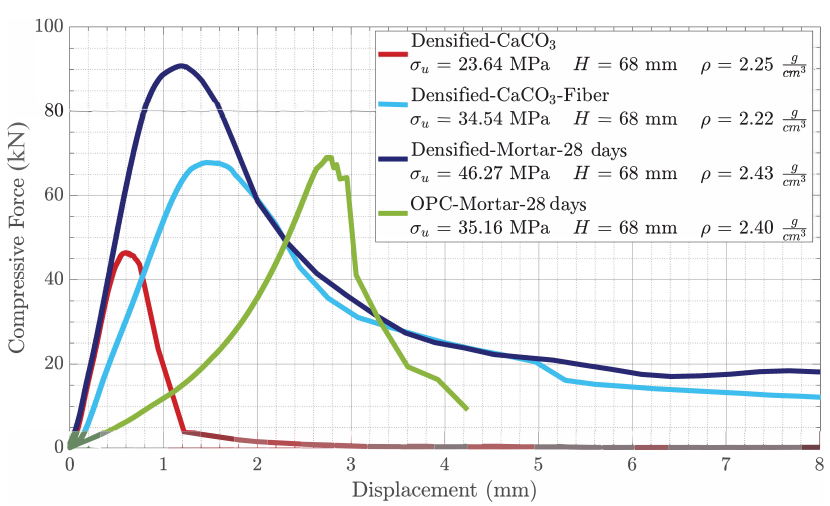

Figure 11. Compressive strength of sintered/densified samples compared to ordinary portland cement mortar. $\sigma_{\mathrm{w}}, H$, and $\rho$ are the ultimate strength, sample height, and density, respectively. All densified samples were prepared by the large mold. All samples had a diameter of $50.8 \mathrm{~mm}$, and the ultimate stresses were calculated by dividing the ultimate force by the cross-sectional area. Mix constituents and sample preparations are shown in Table 2.

alkalinity of the solution ( $1 \mathrm{M})$ is not deleterious to the homopolymer polypropylene fiber utilized.

The combined effect of fiber and partial replacement of $\mathrm{CaCO}_{3}$ by OPC (OPC content of $16.7 \%$ only) in the sintered/ densified mortar sample led to a strength of $46.3 \mathrm{MPa}$, higher than $35.16 \mathrm{MPa}$ strength of the OPC mortar. Scaling up the setup can potentially accommodate larger aggregate sizes from which large precast blocks can be produced.

As a result of its finer and more uniform particle size distribution, PCC exhibits higher potential in terms of regular grain boundary growth under sintering when compared to GCC. Nonetheless, sintered PCC samples showed lower 

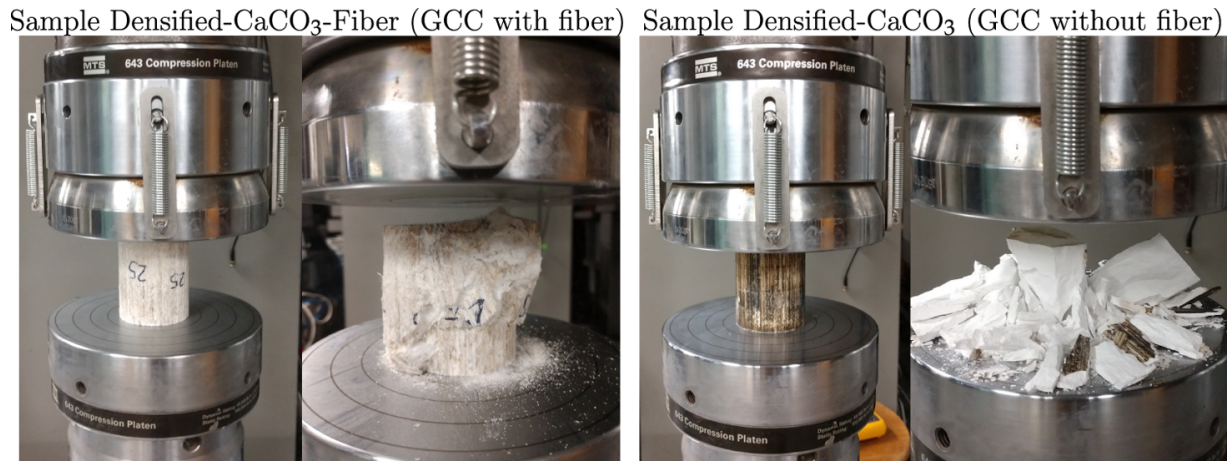

Figure 12. Failure behavior of sintered/densified $\mathrm{CaCO}_{3}$ samples with and without the fiber. Mix constituents and sample preparation are shown in Table 2. The brown coat on the sample on the right is from the release agent oil in the inner face of the mold sleeve tube.

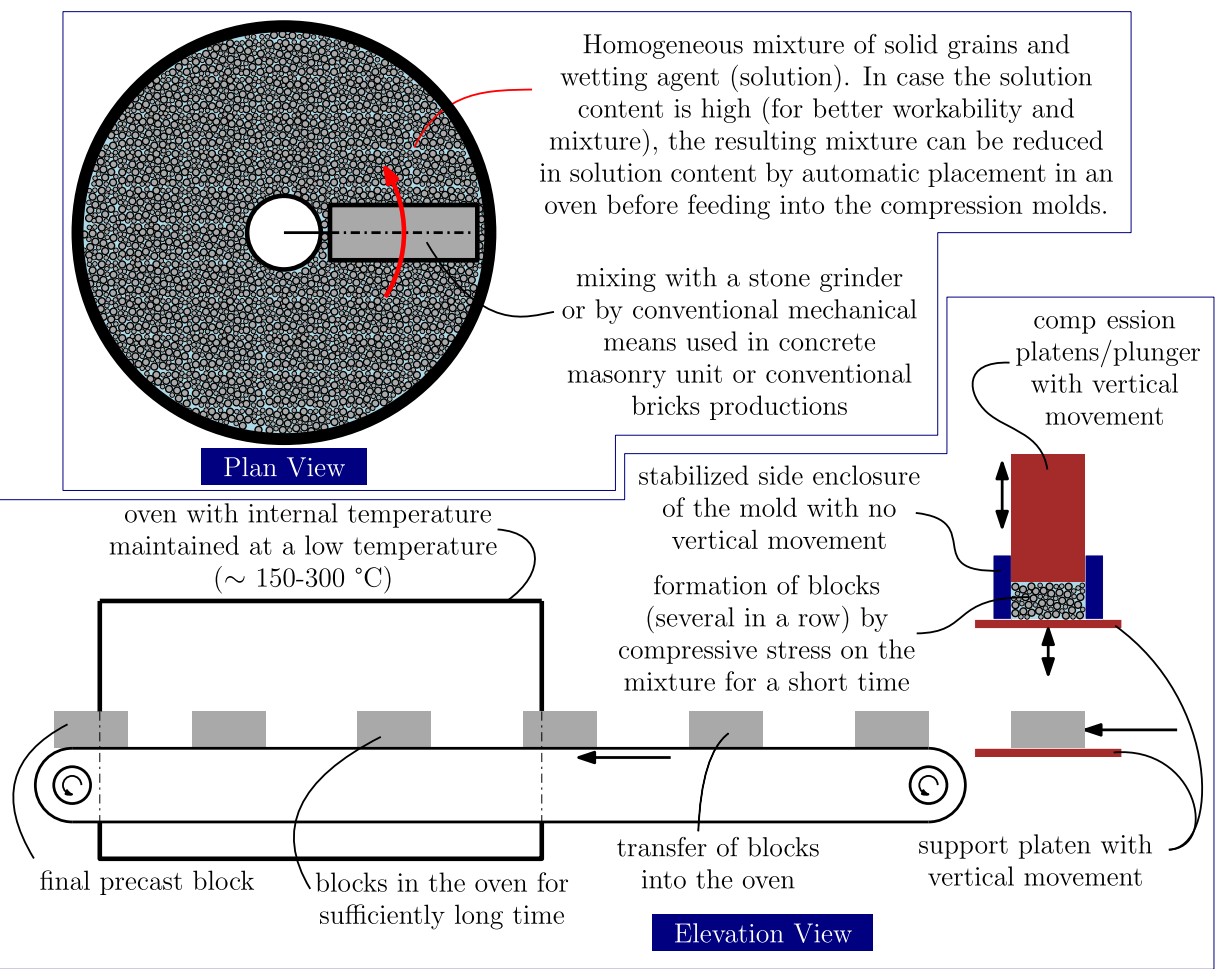

Figure 13. Schematic of one alternative for the large-scale production of construction material blocks through cold sintering.

fracture toughness and structural soundness compared to GCC samples when handling the samples. This is attributed to the inability to achieve homogenous sintering within the PCC samples. An additional contribution to the PCC samples' brittleness might have resulted from small water entrapments originally occupied by the solution, leaving a large number of pores behind upon evaporation, thus lowering the fracture toughness, as reported in the literature. ${ }^{11}$ PCC can, however, be explored when combined with cementitious materials. Higher structural soundness of GCC samples is believed to be caused by their higher compaction due to wider particle size distribution, as reported in the Nitrogen AdsorptionDesorption section.

GCC, by the virtue of its low production cost and desirable sintering/densification observed in the SEM and the porosimetry results, merits further study of its performance with additives. Calcium carbonate can be supplemented with small doses of ordinary cements and mineral additives to further improve the mechanical properties. As in conventional quasibrittle construction materials that often require reinforcements, fibrous reinforcements can enhance the mechanical properties. It was shown that fiber improved the compressive strength of densified GCC, while the addition of both sand as aggregate and cement further enhanced the strength. The goal of the cold sintering is to utilize abundant or low-cost raw materials such as limestone, clay, or calcined clay as the major constituents (as high as 80 weight \%) of the binder. The remaining constituents of the binder such as cement and fiber or reinforcement are expected to give additional structural integrity that might be needed for a specific application such as masonry blocks. It is noted that calcium carbonate presence in the final product must be limited for applications exposed to such gaseous pollutants as sulfur dioxide or nitrogen oxides and acid rain due to their deleterious effect as discussed in the Introduction section. Therefore, incorporation of constituents with higher durability such as clay might be a necessary step to take. Aggregates can be present as the major constituents (as 
high as 80 weight \%) of the block similar to conventional construction materials.

Cold sintering can be used to scale up production of construction material blocks. Ground raw materials for conventional bricks are formed by low compressive stresses and fed into high-temperature kilns. Concrete masonry units (CMUs), however, are formed by similar low compressive stresses but cured at low temperatures since ordinary cement hydration provides binding for CMU constituents. Cold sintering falls between the two in terms of the temperature requirement. Schematic of one alternative for automated production through cold sintering is illustrated in Figure 13. The compressive stress application precedes the heat exposure in this setup, and the sequence appears to be the most economical one. If sufficiently high stresses are applied and suitable wetting agents are used, it is expected that grain boundary growth could still take place despite asynchronous heat and compressive stress exposures. Alternatively, the mold assembly can be placed inside of the oven and simultaneous heat and stress be applied at higher stress dwell times.

\section{CONCLUSIONS}

Cold sintering of commercially available ground and precipitate calcite grains was investigated. Zinc oxide was used for comparison, and cement and fiber were used to evaluate their potential impact on mechanical properties. A small and a large mold were used to compare the effectiveness of the sample preparation and homogeneity of the powders wetted by water or aqueous solutions of $\mathrm{NaOH}, \mathrm{NaCl}$, or acetic acid. $\mathrm{NaOH}$ solution outperformed other wetting agents in sintering of grains of PCC and GCC, whereas acetic acid excelled in sintering of zinc oxide. The small mold was shown to outperform the large mold as the former's sample volume requirement was considerably lower, allowing higher control over sample preparation and homogeneous access of the wetting agents to the solid particles. More sophisticated mixing equipment can facilitate scaling up the compacts.

The superplasticizer helped in the initial mixing of the $\mathrm{CaCO}_{3}$ and $\mathrm{ZnO}$ samples by providing a workable mixture while maintaining the low starting solution-to-solid ratios. This workable mixture needed to be elevated in viscosity nonetheless for it to not escape through the mold gap during the sintering stage. For this purpose, a secondary material is typically needed. This material could range from cementitious materials to aggregates or fibers to clay minerals to supplementary cementing materials such as silica fume. Clay minerals are believed to be particularly promising when combined with calcium carbonate in the preparation of samples through cold sintering for construction material applications.

\section{ASSOCIATED CONTENT}

\section{SI Supporting Information}

The Supporting Information is available free of charge at https://pubs.acs.org/doi/10.1021/acsomega.0c04617.

Cold sintering setup (Figure S1); four grades of calcium carbonate with descending water affinity (Figure S2); typical preparation method of the cold sintering samples (Figure S3); heater assembly utilized in the cold sintering tests (Figure S4); large mold and application of silicone (Figure S5); small mold for application of higher stresses (Figure S6); densified/sintered PCC in the presence of ordinary portland cement with the assistance of $\mathrm{NaOH}$ solution (Figure S7); densified PCC under high stress of $750 \mathrm{MPa}$ with $\mathrm{NaCl}$ solution (Figure S8); densified PCC with $\mathrm{NaCl}$ solution (Figure S9); densified/transformed PCC with acetic acid solution (Figure S10); inefficacy in sintering $\mathrm{ZnO}$ with acetic acid solution prepared by the large mold (Figure S11); and densified GCC (70\%) $\mathrm{ZnO}$ (30\%) prepared by the large mold (Figure S12) (PDF)

\section{AUTHOR INFORMATION}

\section{Corresponding Author}

Aly Said - Department of Architectural Engineering, The Pennsylvania State University, University Park, Pennsylvania

16802, United States; Phone: 814-863-2087;

Email: aly.said@engr.psu.edu

\section{Authors}

Mehrzad Zahabi - McNamara Salvia Structural Engineers, Boston, Massachusetts 02110, United States; 이이.org/ 0000-0002-5844-3790

Ali Memari - Department of Architectural Engineering and Department of Civil and Environmental Engineering, The Pennsylvania State University, University Park, Pennsylvania 16802, United States

Complete contact information is available at:

https://pubs.acs.org/10.1021/acsomega.0c04617

\section{Notes}

The authors declare no competing financial interest.

\section{ACKNOWLEDGMENTS}

The authors thank the support from the Penn State College of Engineering, the Department of Architectural Engineering, and Materials Research Institute.

\section{REFERENCES}

(1) Mehta, K.; Monteiro, P. Concrete: Microstructure, Properties, and Materials; McGraw-Hill Publishing, 2014

(2) Brick Industry Association. Technical Notes on Brick Construction-Manufacturing of Brick; The Brick Industry Association: Reston, VA, 2006; Vol. 9.

(3) Sintered Refractory Bricks by High-Temperature Treatment of Dolomite. http://www.kelsen.es/dolomia_en.html (accessed 202009-20).

(4) Limestone Calcined Clay Cements (LC3). https://www.lc3.ch/ (accessed 2020-09-20).

(5) Avet, F.; Sofia, L.; Scrivener, K. Concrete Performance of Limestone Calcined Clay Cement $\left(\mathrm{LC}^{3}\right)$ Compared with Conventional Cements. Adv. Civ. Eng. Mater. 2019, 8, 275-286.

(6) Schneider, J. Decarbonizing construction through carbonation. Proc. Natl. Acad. Sci. U.S.A. 2019, 12515-12517.

(7) Gartner, E.; Sui, T. Alternative cement clinkers. Cem. Concr. Res. 2018, 114, 27-39.

(8) Concentrating Solar Power Projects. https://solarpaces.nrel.gov/ (accessed 2020-09-20)

(9) Effinger, M.; Tucker, D. Statistical Design Study of Lunar Ceramic; National Aeronautics and Space Administration, George C. Marshall Space Flight Center, 1994; p 108465.

(10) Guo, H.; Baker, A.; Guo, J.; Randall, C. A. Cold Sintering Process: A Novel Technique for Low-Temperature Ceramic Processing of Ferroelectrics. J. Am. Ceram. Soc. 2016, 99, 3489-3507.

(11) Ndayishimiye, A.; Largeteau, A.; Mornet, S.; Duttine, M.; Dourges, M.-A.; Denux, D.; Verdier, M.; Gouné, M.; de Beauvoir, T. H.; Elissalde, C.; et al. Hydrothermal sintering for densification of 
silica. Evidence for the role of water. J. Eur. Ceram. Soc. 2018, 38, 1860-1870.

(12) Guo, J.; Zhao, X.; Herisson De Beauvoir, T.; Seo, J.-H.; Berbano, S. S.; Baker, A. L.; Azina, C.; Randall, C. A. Recent Progress in Applications of the Cold Sintering Process for Ceramic-Polymer Composites. Adv. Funct. Mater. 2018, 28, No. 1801724.

(13) Maria, J.-P.; Kang, X.; Floyd, R. D.; Dickey, E. C.; Guo, H.; Guo, J.; Baker, A.; Funihashi, S.; Randall, C. A. Cold sintering: Current status and prospects. J. Mater. Res. 2017, 32, 3205-3218.

(14) Bouville, F.; Studart, A. R. Geologically-inspired strong bulk ceramics made with water at room temperature. Nat. Commun. 2017, 8, No. 14655.

(15) Yamanaka, S.; Sugawara, Y.; Oiso, T.; Fujimoto, T.; Ohira, Y.; Kuga, Y. Phase transformation of mesoporous calcium carbonate by mechanical stirring. CrystEngComm 2015, 17, 1773-1777.

(16) Dachille, F.; Roy, R. High-pressure phase transformations in laboratory mechanical mixers and mortars. Nature 1960, 186, 34.

(17) Burns, J. H.; Bredig, M. Transformation of calcite to aragonite by grinding. J. Chem. Phys. 1956, 25, 1281.

(18) Chaim, R.; Levin, M.; Shlayer, A.; Estournès, C. Sintering and densification of nanocrystalline ceramic oxide powders: a review. $A d v$. Appl. Ceram. 2008, 107, 159-169.

(19) Kang, X.; Floyd, R.; Lowum, S.; Cabral, M.; Dickey, E.; Maria, J.-P. Mechanism studies of hydrothermal cold sintering of zinc oxide at near room temperature. J. Am. Ceram. Soc. 2019, 102, 4459-4469.

(20) Frear, G. L.; Johnston, J. The solubility of calcium carbonate (calcite) in certain aqueous solutions at $25^{\circ}$ C. J. Am. Chem. Soc. 1929, 51, 2082-2093.

(21) De Silva, P.; Bucea, L.; Sirivivatnanon, V.; Moorehead, D. R. Carbonate binders by "cold sintering" of calcium carbonate. J. Mater. Sci. 2007, 42, 6792-6797.

(22) Ito, J.; Matsushima, Y.; Unuma, H.; Horiuchi, N.; Yamashita, K.; Tajika, M. Preparation and properties of pressureless-sintered dense calcite ceramics. Mater. Chem. Phys. 2017, 192, 304-310.

(23) Acid Rain Program. https://www.epa.gov/acidrain/acid-rainprogram (accessed 2020-09-20).

(24) Charola, A. E.; Ware, R. Acid deposition and the deterioration of stone: a brief review of a broad topic. Geol. Soc., Spec. Publ. 2002, 205, 393-406.

(25) Funahashi, S.; Guo, J.; Guo, H.; Wang, K.; Baker, A. L.; Shiratsuyu, K.; Randall, C. A. Demonstration of the cold sintering process study for the densification and grain growth of $\mathrm{ZnO}$ ceramics. J. Am. Ceram. Soc. 2017, 100, 546-553.

(26) Lowum, S.; Floyd, R.; Bermejo, R.; Maria, J.-P. Mechanical strength of cold-sintered zinc oxide under biaxial bending. J. Mater. Sci. 2019, 54, 4518-4522.

(27) Ellis, A. The solubility of calcite in sodium chloride solutions at high temperatures. Am. J. Sci. 1963, 261, 259-267.

(28) Mucci, A. The solubility of calcite and aragonite in seawater at various salinities, temperatures, and one atmosphere total pressure. Am. J. Sci. 1983, 283, 780-799.

(29) Lister, T. Sodium Carbonate: A Versatile Material; Royal Society of Chemistry, 2000.

(30) Yamasaki, N.; Tang, W.; Ke, J. Low-temperature sintering of calcium carbonate by a hydrothermal hot-pressing technique. J. Mater. Sci. Lett. 1992, 11, 934-936.

(31) Lin, P.-W.; Shen, P. Onset sintering-coarsening-coalescence kinetics of calcite powders. J. Eur. Ceram. Soc. 2013, 33, 3265-3272.

(32) Gebauer, D.; Liu, X.; Aziz, B.; Hedin, N.; Zhao, Z. Porous tablets of crystalline calcium carbonate via sintering of amorphous nanoparticles. CrystEngComm 2013, 15, 1257-1263.

(33) Scrivener, K.; Snellings, R.; Lothenbach, B. A Practical Guide to Microstructural Analysis of Cementitious Materials; CRC Press, 2016.

(34) Hewlett, P.; Liska, M. Lea's Chemistry of Cement and Concrete; Butterworth-Heinemann, 2019. 